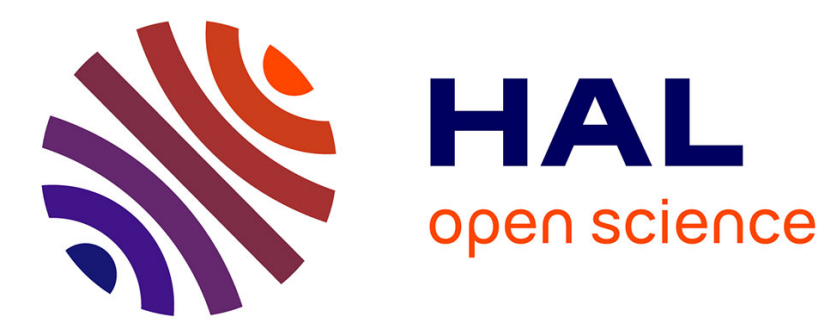

\title{
Le moment politique de l'économie russe
}

Julien Vercueil

\section{To cite this version:}

Julien Vercueil. Le moment politique de l'économie russe. Revue Défense Nationale, 2015, 783, pp.91-97. halshs-01422246

\section{HAL Id: halshs-01422246 \\ https://shs.hal.science/halshs-01422246}

Submitted on 24 Dec 2016

HAL is a multi-disciplinary open access archive for the deposit and dissemination of scientific research documents, whether they are published or not. The documents may come from teaching and research institutions in France or abroad, or from public or private research centers.
L'archive ouverte pluridisciplinaire HAL, est destinée au dépôt et à la diffusion de documents scientifiques de niveau recherche, publiés ou non, émanant des établissements d'enseignement et de recherche français ou étrangers, des laboratoires publics ou privés.

\section{(이) $\$$}

Distributed under a Creative Commons Attribution - NonCommercial - NoDerivatives 44.0 


\title{
Le moment politique de l'économie russe
}

\author{
Julien Vercueil \\ Centre de Recherches Europes - Eurasie, \\ INALCO
}

Les trajectoires économiques sont le fruit d'interactions entre les politiques publiques et les stratégies privées. En règle générale, les premières tiennent compte de leur influence sur les secondes, et réciproquement. Cette réciprocité fait que ces interactions sont le plus souvent régulatrices : elles sont en mesure de produire des trajectoires socioéconomiques relativement stables dans le temps. Une décision politique malheureuse peut être corrigée au vu de ses conséquences sur l'économie ou des protestations qu'elle suscite ; ou bien, certaines stratégies d'acteurs privés sont contrecarrées - voire interdites - par la puissance publique, qui estime qu'elles font courir des risques excessifs à la collectivité. Dans ces deux cas, une forme de régulation a lieu, qui permet au système socio-économique de continuer de fonctionner dans un environnement dont les changements sont graduels et relativement prévisibles.

Quelquefois en revanche, le politique devient hégémonique. Le système social et le système économique sont sommés de s'adapter, au moins pour un temps, aux politiques menées. Nous qualifierons ce type de période de "moment politique".

Depuis mars 2014, la Russie est entrée dans un "moment politique". Les décisions d'annexer la Crimée, puis de soutenir les séparatistes du Donbass ont constitué des ruptures politiques majeures pour le pays, nombre d'analyses en conviennent. Ce qui est moins souligné en revanche, c'est que cette rupture est à l'origine de secousses économiques considérables. Les pertes économiques subies par la grande majorité de la population russe ne peuvent pas être compensées par l'amélioration de la situation de quelques acteurs bien placés. Pourtant, le système économique et social s'est jusqu'ici accommodé de cette nouvelle donne. C'est précisément cet accommodement qui signe le "moment politique" vécu actuellement en Russie.

L'analyse que nous proposons dans cet article tient en trois temps : dans un premier temps, nous résumerons les canaux par lesquels la nouvelle donne géopolitique a affecté l'économie. Nous dresserons ensuite ses perspectives d'évolution, en supposant que rien ne vienne bouleverser le contexte actuel. Enfin, nous analyserons les chances de sortie $\mathrm{du}$ "moment politique" à moyen terme.

\section{Le moment politique et son impact économique : printemps 2014 - été 2015}

Les commentaires ont beaucoup insisté sur le rôle des sanctions occidentales dans le décrochage de l'économie russe à partir du deuxième semestre 2014. En réalité, ce rôle est plus modeste qu'il n'y paraît. Certes, les principales banques et entreprises énergétiques russes se sont retrouvées brusquement dans l'impossibilité de recourir au marchés des capitaux pour renouveler leur dette en devises, ce qui a bloqué de nombreux projets d'investissement. Mais les analyses économiques sérieuses reconnaissent l'effet macroéconomique de ce choc extérieur a été mineur (Ivanter 
(2015), Central Bank of Russia (2015)). Deux autres facteurs expliquent l'essentiel de la dégradation de la conjoncture.

Le premier est l'élévation de l'incertitude géopolitique. A partir de l'annexion de la Crimée, la Russie est apparue à nombre d'acteurs économiques internationaux comme un pays où les décisions politiques peuvent remettre en cause n'importe quelle règle préalablement établie. La frontière russo-ukrainienne est devenue une zone de guerre et non plus une zone d'échanges. La radicale incertitude créée par cette nouvelle configuration a eu des effets sur les marchés financiers en Russie (qui ont chuté au moment même où les troupes russes faisaient irruption en territoire jusque là ukrainien), sur le rouble (dont l'effritement par rapport à l'euro et au dollar s'est accéléré) et surtout sur l'image de la région auprès des investisseurs russes et étrangers. Le rythme des sorties de capitaux a plus que doublé en 2014, tandis que la plupart des décisions d'investissement étaient reportées sine die. L'impact immédiat du moment politique sur la situation financière de la Russie a été tel que pour l'année 2014, il est possible de retracer, semaine après semaine, les inflexions de plusieurs indicateurs macro-financiers clés de la Russie en suivant le déroulement des événements diplomatiques et militaires sur le terrain (Vercueil, 2014b).

Le deuxième facteur est la chute des prix mondiaux des hydrocarbures. A partir de l'été 2014, elle a tari les sources de devises des entreprises exportatrices, de la Banque centrale de Russie et de l'État. Les anticipations sur le taux de change du rouble sont devenues défavorables, compte tenu du fait que plus de $70 \%$ des exportations de la Russie sont constituées de matières premières. Dans le même temps, la Banque centrale de Russie poursuivi la transition annoncée de sa politique monétaire vers le ciblage d'inflation, surestimant la confiance des agents économiques envers le rouble. Après deux mois d'érosion accélérée, le taux de change de la monnaie russe a brusquement dévissé de $20 \%$ le 16 décembre. Cet effondrement a provoqué une nouvelle déflagration financière à l'échelle du pays, obligeant la Banque centrale à dépenser sur le marché des changes une part importante de ses réserves, fragilisant davantage la situation des agents endettés en dollars, accélérant l'inflation dont la réduction constituait pourtant le principal objectif des autorités monétaires, sapant enfin la confiance des particuliers et des entreprises dans la capacité des autorités à stabiliser la situation économique.

A ces deux facteurs principaux peut être ajouté l'embargo de la Russie sur les importations de certains produits agricoles en provenance des pays ayant appliqué les sanctions à son encontre. Le temps que les agents économiques s'adaptent à cette nouvelle décision, la rupture des circuits d'approvisionnement a produit des augmentations de prix dans tous les canaux de distribution. Certains produits n'ont pas trouvé de substitut, ce qui a entraîné une réduction de la variété et de la qualité moyenne des produits disponibles. Enfin, des moyens informels de contournement de l'embargo se sont mis en place, créant à la fois des brèches dans la crédibilité de ces mesures, un surcoût lié à la surveillance des flux, des occasions supplémentaires de trafics et de corruption et des sources de frictions et de conflits avec les partenaires traditionnels de la Russie que sont la Biélorussie et le Kazakhstan, lesquels ne souhaitent ni participer aux sanctions occidentales, ni appliquer l'embargo russe. Toutefois, l'embargo russe sur a eu sur la conjoncture du pays des conséquences qui ne sont pas du même ordre de grandeur que celles des tensions géopolitiques et de la chute des cours du pétrole. 
Confronté à ces chocs, l'économie de la Russie, déjà affaiblie et en phase de ralentissement (Vercueil, 2014a) n'a pas pu tenir longtemps sur une trajectoire positive. En 2014, elle s'est enfoncée dans la stagflation. La déflagration financière de décembre 2014 a transformé cette trajectoire en récession dès le premier semestre 2015 : entre janvier et mai, la chute de la production manufacturière a atteint 4,1\% en rythme annuel, le commerce de détail de 7,7 \%, les exportations de 29,3\% et les importations de 38,3\%. L'augmentation du taux d'intérêt directeur de la Banque centrale, relevé à $17 \%$ en décembre, a réussi à stopper la spéculation mais a également aggravé la détérioration des conditions d'investissement, sans empêcher l'inflation d'accélérer sous l'effet de l'effondrement du rouble. Au premier semestre 2015 l'indice des prix à la consommation a atteint un rythme annuel de $16 \%$, contribuant à la chute les revenus réels de la population de près de $9 \%$.

\section{La trajectoire actuelle de l'économie russe}

Confrontées à cette situation qu'elles n'avaient pas anticipée, les autorités ont pris des mesures dans l'objectif de limiter la chute de la demande globale. Dans le domaine monétaire, les taux d'intérêt ont été peu à peu réduits après le pic de décembre, pour s'établir à $11 \%$ en août 2015. Le gouvernement a soutenu les établissements bancaires en difficulté, ce qui a permis d'éviter une contagion de la crise financière. Dans le même temps, la profonde chute du rouble a provoqué une contraction des importations, qui a joué un rôle d'amortisseur de la crise productive. Enfin, sur le plan budgétaire des mesures contra-cycliques ont été prises : après une période d'hésitations (en février 2015 le gouvernement prévoyait encore des coupes de $10 \%$ dans les dépenses budgétaires), le gouvernement a finalement décidé de laisser filer le déficit budgétaire pour éviter d'ajouter à la chute de la demande des ménages et des entreprises celle des commandes publiques (graphique 1). 


\section{Graphique 1. Dépenses et recettes du budget \\ de la Fédération de Russie, \% du PIB \\ (2008-2015, moyennes mobiles trimestrielles)}

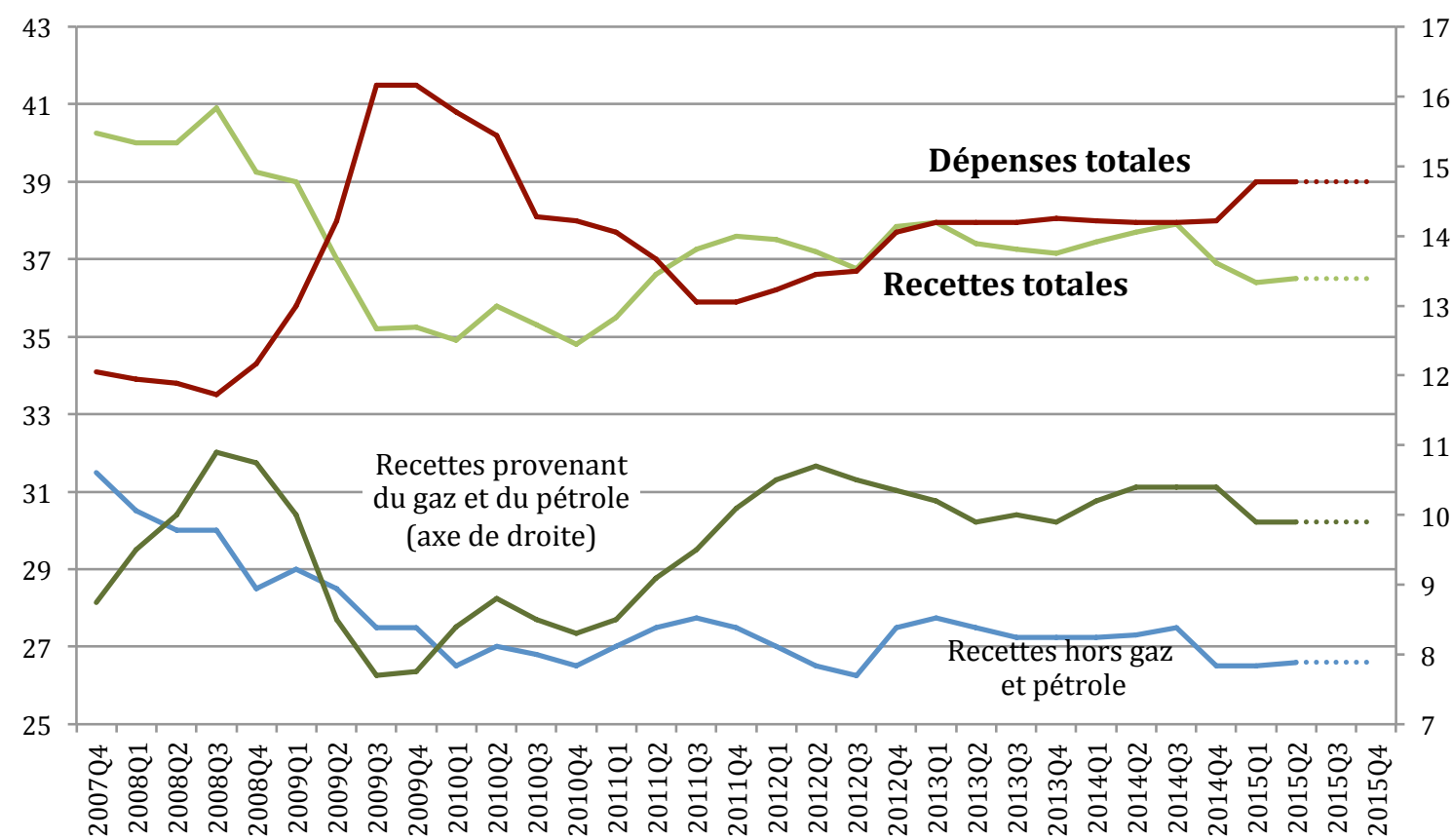

Source : Banque centrale de Russie. Pointillés : prévisions officielles. Q : trimestre

La combinaison de la chute des revenus fiscaux et de l'avancement de certains programmes de dépense, notamment en matière de défense, devrait conduire le gouvernement à enregistrer un déficit budgétaire de l'ordre de 3,7 \% du PIB en 2015. Pour limiter l'endettement extérieur, il mobilisera le Fonds de réserve à hauteur de 2000 milliards de roubles, soit environ 2,8 \% du PIB. En outre, les investissements contra-cycliques dans les projets d'infrastructure réalisés à partir du Fonds national de bien être (initialement destiné à garantir le niveau des retraites futures) pourraient atteindre 2 à 2,4 milliers de milliards de roubles supplémentaires en 2015-2018 (Central Bank of Russia, 2015), ce qui représenterait une contribution de 0,8 à 0,9\% du PIB par an pendant quatre ans. Mises bout à bout, ces mesures correspondraient à une impulsion budgétaire totale comprise entre 6 et $8 \%$ du PIB, soit plus de la moitié de celle de 2009. Mais l'orientation de la politique budgétaire pourrait se retourner à moyen terme. Les objectifs annoncés par le Ministère des finances en matière de réduction du déficit budgétaire doivent se traduire en effet par une compression de $5 \%$ des dépenses publiques en termes réels durant les deux prochaines années ${ }^{1}$. Compte tenu de la trajectoire macro-économique actuelle de la Russie, ces objectifs ont quelque chance d'être révisés.

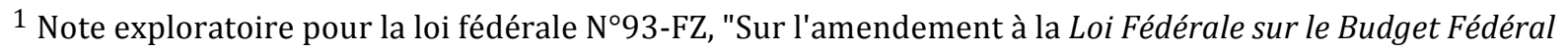
de 2015 et la période planifiée de 2016-2017", 20 avril 2015.
} 
Graphique 2. Le PIB de la Russie en roubles, en dollars et en euros (2013-2015)

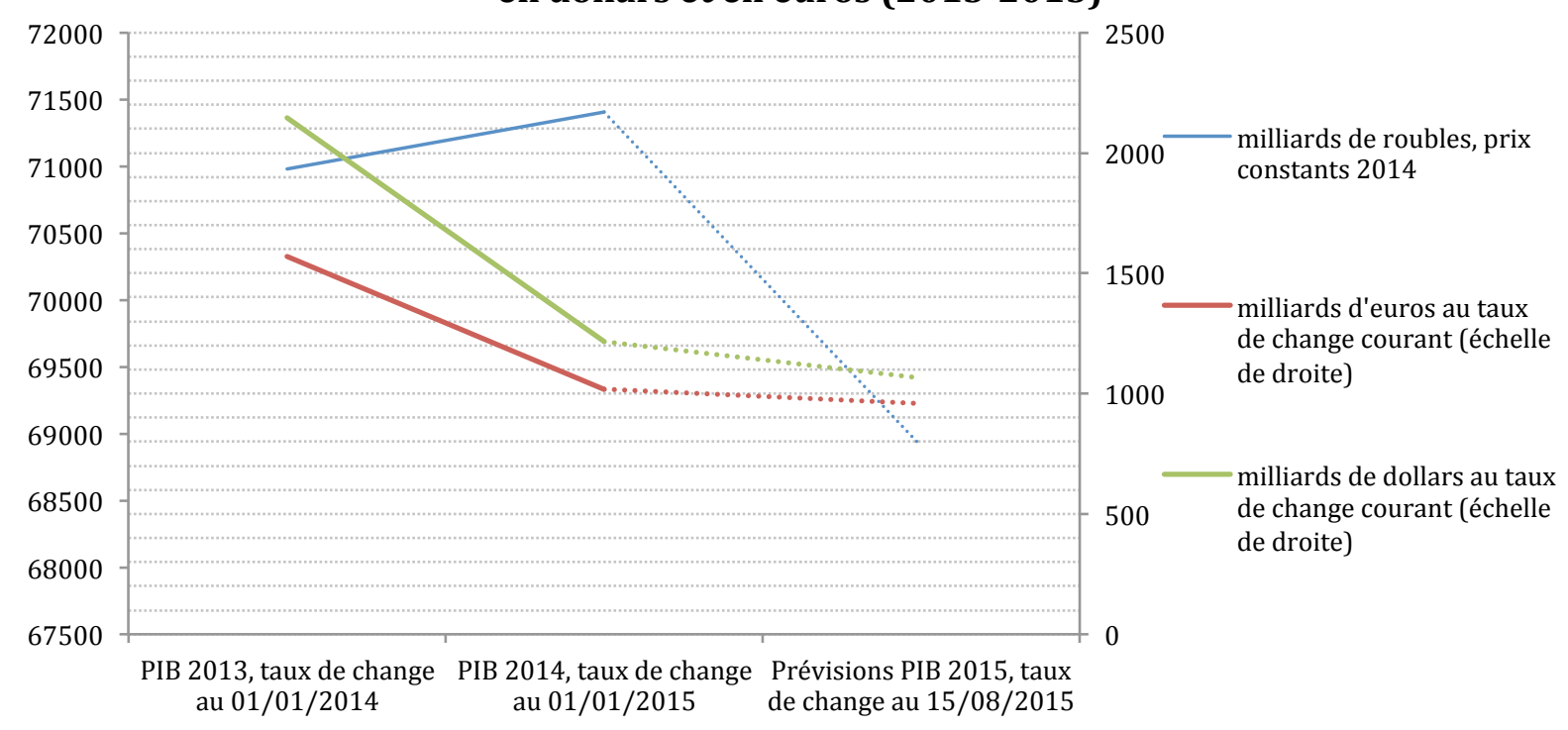

Source : élaboration de l'auteur à partir de données Rosstat et Banque centrale de Russie (2015).

Pointillés : prévisions

Les efforts des autorités pour relancer l'activité, n'empêchent pas le climat géopolitique et la chute des recettes d'exportations de tirer la conjoncture vers le bas. La moyenne des prévisions de croissance des instituts de conjoncture pour 2015 se situe actuellement aux alentours de $-3,5 \%$, soit le plus mauvais chiffre depuis 2009. En termes de valeur internationale du PIB, la chute cumulée depuis fin 2013 au taux de change courant s'établissait fin août 2015 à plus de 1000 milliards de dollars (Graphique 2). La crise a d'abord été financière, pour se transformer en crise économique à partir de la fin de l'année 2014. Pour 2016, les projections disponibles envisagent une stabilisation avec une croissance comprise entre 0 et $1 \%$ (IMF, 2015). Au total, la Russie est le pays des BRICS qui a connu le plus violent retournement de conjoncture entre 2008 et 2015 et dont les performances macroéconomiques sont les plus faibles depuis 2013. Le moment politique a bien eu un coût très lourd pour l'économie et la société russes.

\section{Sortir du moment politique?}

Les conditions ne sont pas réunies actuellement en Russie pour sortir du moment politique. L'opinion publique a été galvanisée par l'annexion de la Crimée et ce qui est désormais présenté et perçu comme une résistance héroïque du peuple russe aux agressions occidentales à son endroit. De ce point de vue, les sanctions mises en place par la plupart des pays de l'OCDE à l'encontre de la Russie ont été politiquement contreproductives ${ }^{2}$.

Deux facteurs plus structurels pourraient toutefois contribuer à engager une modification de la position russe sur le conflit en Ukraine, susceptible de rapprocher le pays de l'Union Européenne.

\footnotetext{
2 Voir par exemple J. Vercueil, "Avis de gros temps sur l'économie russe", Le Monde Diplomatique, février 2015.
} 
- D'un côté, la perspective des effets à moyen terme de l'isolement de la Russie des circuits internationaux de capitaux et de technologies-clés (énergie et armement) apparaît peu à peu aux dirigeants russes. Passé le moment d'enthousiasme lié à un "pivot" supposé vers l'Asie, les conséquences d'un développement de relations économiques exclusives avec la Chine commencent à apparaître dans toutes leurs dimensions : avec des prix du gaz tirés vers le bas dans le contrat de mai 2014 et l'acquisition de parts significatives de gisements d'hydrocarbures, la Chine a bénéficié de sa relation asymétrique avec la Russie et risque désormais de considérer son partenaire, désormais huit fois plus faible qu'elle économiquement, comme un simple réservoir de matières premières. Par sa puissance commerciale, sa capacité en matière de génie civil, ses technologies, ses ressources financières et son insatiable appétit énergétique, elle lui fait désormais concurrence dans son jardin historique d'Asie centrale. Isolée, la Russie n'est tout simplement pas de taille à rivaliser avec la puissance chinoise.

- D'un autre côté, l'Union Économique Eurasienne, instituée à marche forcée durant les cinq dernières années, ne peut constituer ni un marché ni un fournisseur de substitution pour la Russie. Elle ne représente pas $8 \%$ de son commerce extérieur, tandis que l'Union Européenne, malgré les sanctions et l'embargo, continue de peser pour près de moitié dans les échanges extérieurs russes. Surtout, le conflit en Crimée et au Donbass a débouché sur la perte de l'objectif principal de l'Union pour la Russie : l'intégration de l'Ukraine, qui par ses liens historiques, culturels et économiques avec elle était appelé à devenir son principal partenaire dans cet ensemble. Au lieu de cela, les dirigeants ukrainiens se considèrent désormais en conflit armé avec la Russie, tandis que l'économie nationale - en particulier le Donbass russophone - est dévastée.

Pour l'instant, aucun argument économique n'a pu infléchir la ligne définie en mars 2014. Le moment politique se prolonge. En l'absence de perspective d'alternance politique, ce sont les dirigeants actuels qu'il faut convaincre de la nécessité d'en sortir rapidement. Les pertes engendrées par les tensions avec l'Occident et le conflit en Ukraine sont considérables pour le pays, mais peu prises en compte. De même, les gains économiques d'une sortie de crise dans la région sont rarement évoqués. Ils peuvent pourtant constituer un point d'appui précieux pour ceux des protagonistes qui souhaitent hâter la fin du moment politique en Russie. 


\section{Références :}

Central Bank of Russia (2015) : Monetary policy Report, №2, June 2015. www.cbr.ru

IMF (2015) : "IMF Country Report No. 15/211. Russian Federation". Washington, D. C. : International Monetary Fund, August 2015.

Ivanter V. (2015) : "L'économie russe : situation actuelle et perspectives", communication au Séminaire Franco-Russe CEMI-IPEN ASR. Paris : EHESS, 22 juin 2015.

Vercueil, J. (2014a) : “L'économie russe en 2013 : les limites du modèle de croissance sont atteintes", in Dubien A. (Dir.), Russie 2014. Regards de l'observatoire franco-russe. Moscou et Paris : CCIFR - Les éditions du cherche midi, 2014, p. 19-30.

Vercueil, J. (2014b) : "L'économie russe et les sanctions. Une évaluation des conséquences du conflit ukrainien $»$. Note de l'observatoire franco-russe, $n^{\circ} 9$, novembre 2014. www.obsfr.ru/fileadmin/Policy_paper/PP9_FR.pdf / 